\title{
Bundles of $(p, A)$-covelocities and $(p, A)$-jets
}

\author{
Jiřr Tomáš
}




\title{
BUNDLES OF $(p, A)$-COVELOCITIES AND $(p, A)$-JETS
}

\author{
JIŘÍ TOMÁŠ
}

\begin{abstract}
Let $M$ be an $m$-dimensional manifold, $P^{r} M$ the frame bundle, $A=\mathbb{D}_{k}^{r} / I=\mathbb{R} \oplus$ $N_{A}$ a Weil algebra and $p: G_{k}^{r} \rightarrow$ Aut $A$ a Lie group homomorphism. For a Lie subgroup $G_{p, k}^{r} \subseteq G_{k}^{r}$ and for $m \geq k$, we define the concept of a $(p, A)$-covelocity extending restrictions $T_{x}^{A} f_{\mid \operatorname{Orb}\left(G_{p, k}^{r}, j^{A} \varphi_{x}\right)}$ of $A$-covelocities to $G_{p, k}^{r}$-orbits with respect to the left action $\ell: G_{m}^{r} \times$ $N_{A} \rightarrow N_{A}$ determined by $p$. Further, we introduce bundle functors $J_{p}^{A}$ of $(p, A)$-jets defined on $\mathcal{M} f_{m} \times \mathcal{M} f$ and give their geometrical description
\end{abstract}

2000 Mathematics Subject Classification: Primary 58A20, 58A32, Secondary 58D15

Keywords: $r$-jet, bundle functor, natural bundle, Weil functor, Lie group, jet group

\section{Preliminaries}

We give a contribution concerning the geometry of Weil functors and their morphisms. The starting points are the concepts of an $r$-jet, a jet space $J^{r}(M, N)$, and a bundle functor, all defined in [9]. As usual, we denote by $M$ an $m$-dimensional manifold and by $N$ a smooth manifold. All manifolds are considered to be $\mathrm{C}^{\infty}$-manifolds. We denote by $\mathcal{M} f_{m}$ the category of $m$-dimensional manifolds with local diffeomorphisms, by $\mathcal{M} f$ the category of smooth manifolds and smooth maps and by $\mathcal{F} \mathcal{M}$ the category of fibered manifolds with smooth fibered maps. Analogously, we denote by $\mathcal{F} \mathcal{M}_{m}$ the category of fibered manifolds with $m$-dimensional bases together with fibered maps covering local diffeomorphisms. Recall that a bundle functor defined on the category $\mathcal{M} f_{m}$ is said to be a natural bundle [9]. Further, we recall the jet functor defined on the category $\mathcal{M} f_{m} \times \mathcal{M} f$ which assigns the space of $r$-jets $J^{r}(M, N)$ of smooth maps $M \rightarrow N$ to any pair $(M, N) \in \operatorname{Obj}\left(\mathcal{M} f_{m} \times \mathcal{M} f\right)$ and the map $J^{r}(g, h): J^{r}\left(M_{1}, N_{1}\right) \rightarrow J^{r}\left(M_{2}, N_{2}\right)$ defined by $j_{x}^{r} f \mapsto j_{f(x)}^{r} h \circ j_{x}^{r} f \circ\left(j_{x}^{r} g\right)^{-1}$ to any pair $(g, h) \in \operatorname{Morph}\left(\mathcal{M} f_{m} \times \mathcal{M} f\right)$.

Among bundle functors, we recall the product preserving bundle functors defined on the category $\mathcal{M} f$. The classical result by Kainz, Michor and others [6,7,9] reads that such functors coincide with Weil functors. Denoting them by $T^{A}$, we involve the associated Weil algebras to their notation. The restriction of a Weil functor to $\mathcal{M} f_{m}$ is

This work was supported by the project No. FCH-S-13-1878, Specific Research at Universities, Ministry of Education, Youth and Sports of the Czech Republic. 
said to be a Weil bundle. There are many authors studying their geometry, e. g., Kolár, Mikulski, Shurygin, Bushueva and many others ([5, 8-10, 14, 15]). Further, there are authors applying and connecting Weil functors with problems from the theory of Lie groups, e.g., Alonso, Muriel, and Muñoz Rodriguez [1,2,12]. Further, there are papers applying Weil functors in the theory of jets, e. g., Kureš [11], who also studies their applications in the theoretical mechanics.

From the algebraic point of view, Weil functors are studied by Bertram (e.g. [3, 4]) who generalizes their definition substituting general fields and rings for reals in the definition of the associated Weil algebra. Weil functors are also applied in the research of geometrical categories (e. g., Nishimura [13]).

Let us resume briefly the elementary concepts of the Weil theory. Consider the algebra $\varepsilon(k)$ of germs of smooth functions defined on $\mathbb{R}^{k}$ at zero. A Weil algebra can be defined either as $A=\mathbb{R} \oplus N_{A}$ for the ideal $N_{A}$ of nilpotent elements (the so called nilpotent ideal) or as a quotient $A=\varepsilon(k) / I$ by an ideal $I$ of finite codimension. It can also be defined as a quotient $A=\mathbb{D}_{k}^{r} / J$ of the so called jet algebra $\mathbb{D}_{k}^{r}$ or, in other words, as the algebra of polynomials of $k$ indeterminates of order at most $r$ factorized by some of its ideal $J$. Finally, we define $\operatorname{width}(A) \operatorname{as} \operatorname{dim}\left(N_{A} / N_{A}^{2}\right)$ and height $(A)$ as the minimal $r$ for which $A=\mathbb{D}_{k}^{r} / J$.

We apply the covariant approach to the definition of Weil functors. This comes out from the $I$-factorization of germs as follows. Two germs germ $\operatorname{gen}_{0} g: \mathbb{R}_{0}^{k} \rightarrow M$ and germ $_{0} h: \mathbb{R}_{0}^{k} \rightarrow M$ taking the same value $x$ at $0 \in \mathbb{R}^{k}$ are said to be $I$-equivalent if and only if germ $_{x} \gamma \circ$ germ $_{0} g-$ germ $_{x} \gamma \circ$ germ $_{0} h \in I$ for any function $\gamma: M \rightarrow \mathbb{R}$ defined near $x$. Classes of such equivalence relation denoted by $j^{A} g$ form the space $T^{A} M$. For a smooth map $\varphi: M \rightarrow N$, the map $T^{A} \varphi: T^{A} M \rightarrow T^{A} N$ is defined by $T^{A} \varphi\left(j^{A} g\right):=j^{A}(\varphi \circ g)$.

We recall that natural transformations $\tilde{t}_{M}: T^{B} M \rightarrow T^{A} M$ bijectively correspond to homomorphisms $t: B \rightarrow A$ and consequentely to the so-called $B$-admissible $A$ velocities defined in [8] as follows. Let $A=\varepsilon(k) / I$ and $B=\varepsilon(p) / J$ be Weil algebras considered as quotiens of germ algebras. For a smooth map $f: \mathbb{R}_{0}^{k} \rightarrow$ $\mathbb{R}_{0}^{p}$ an $A$-velocity $j^{A} f$ is said to be $B$-admissible if and only if the condition of admissibility

$$
\operatorname{germ}_{0} \varphi \in J \Rightarrow \operatorname{germ}_{0}(\varphi \circ f) \in I
$$

is satisfied for every smooth function $\varphi: \mathbb{R}^{p} \rightarrow \mathbb{R}$. Further, every $B$-admissible $A$ velocity $j^{A} f$ is assigned bijectively a natural transformation $\tilde{t}_{M}: T^{B} M \rightarrow T^{A} M$ defined by $\tilde{t}_{M}\left(j^{B} \varphi\right)=t_{M}^{j^{A} f}\left(j^{B} \varphi\right)=j^{A}(\varphi \circ f)$. It follows that automorphisms of $A$ are determined by reparametrizations of indeterminates $\tau_{1}, \ldots, \tau_{k} \in \mathbb{D}_{k}^{r}$ satisfying the admissibility condition (1.1).

Let $G_{m}^{r}=\operatorname{inv} J_{0}^{r}\left(\mathbb{R}^{m}, \mathbb{R}^{m}\right)_{0}$ with the composition of jets be a general jet group. Recall the identification of $G_{m}^{r}$ with the group $\operatorname{Aut}\left(\mathbb{D}_{m}^{r}\right)$ of Weil algebra automorphisms assigning to every $j_{0}^{r} g \in G_{m}^{r}$ the automorphism of $\mathbb{D}_{m}^{r}$ defined by $j_{0}^{r} \eta \mapsto$ 
$j_{0}^{r} \eta \circ\left(j_{0}^{r} g\right)^{-1}$ for any $j_{0}^{r} \eta \in \mathbb{D}_{m}^{r}$. For a Weil algebra $A=\mathbb{D}_{m}^{r} / I$ and the projection homomorphism $p: \mathbb{D}_{m}^{r} \rightarrow A$, Alonso defined the subgroup $G^{A} \subseteq G_{m}^{r} \simeq \operatorname{Aut}\left(\mathbb{D}_{m}^{r}\right)$ and its normal subgroup $G_{A}$ ([1]) as follows

$$
\begin{aligned}
G_{A} & =\left\{j_{0}^{r} g \in G_{m}^{r} ; p \circ j_{0}^{r} g=p\right\}, \\
G^{A} & =\left\{j_{0}^{r} g \in G_{m}^{r} ; \operatorname{Ker}\left(p \circ j_{0}^{r} g\right)=\operatorname{Ker}(p)\right\} .
\end{aligned}
$$

He also proved the identification $G^{A} / G_{A} \simeq$ Aut $A$ of Lie groups.

In [16], we have defined the spaces $T^{A *} M$ of $A$-covelocities on $M$ by $T^{A *} M=$ $\left\{T_{x}^{A} f: T_{x}^{A} M \rightarrow T_{0}^{A} \mathbb{R} \simeq N_{A} ; x \in M\right\}$ and for local diffeomorphisms $g: M \rightarrow N$ the maps $T^{A *} g: T^{A *} M \rightarrow T^{A *} N$ by $T^{A *} g\left(T_{x}^{A} f\right)=T_{x}^{A} f \circ\left(T_{x}^{A} g\right)^{-1}$. For $A=\mathbb{D}_{k}^{r}$, we write $T_{k}^{r *} M$ and $T_{k}^{r *} g$ (see [9]). In [16], we have proved the following statement.

(a) Let $A=\mathbb{D}_{k}^{r} / I$ and $m=\operatorname{dim} M \geq k$. Then, the spaces $T^{A *} M$ with the maps $T^{A *} g$ form the natural bundle $P^{r} M\left[N_{m}^{r}, \ell\right]$ identified with $T_{m}^{r *} M$ where $N_{m}^{r}$ is the nilpotent ideal of $\mathbb{D}_{m}^{r}$ and $\ell: G_{m}^{r} \times N_{m}^{r}$ is the left action defined by $\ell\left(j_{0}^{r} g, j_{0}^{r} \eta\right)=j_{0}^{r} \eta \circ\left(j_{0}^{r} g\right)^{-1}$

For any $(M, N) \in \operatorname{Obj}\left(\mathcal{M} f_{m}\right) \times \operatorname{Obj}(\mathcal{M} f)$, define $J^{A}(M, N)=\left\{T_{x}^{A} f ; f:\right.$ $M \rightarrow N\}$. For a local diffeomorphism $g: M_{1} \rightarrow M_{2}$ and a smooth map $h: N_{1} \rightarrow$ $N_{2}$, define the map $J^{A}(g, h): J^{A}\left(M_{1}, N_{1}\right) \rightarrow J^{A}\left(M_{2}, N_{2}\right)$ by

$$
J^{A}(g, h)\left(T_{x}^{A} f\right)=T_{f(x)}^{A} h \circ T_{x}^{A} f \circ\left(T_{x}^{A} g\right)^{-1} .
$$

Then, it holds

(b) For $m \geq k$ and $A=\mathbb{D}_{k}^{r} / I$ the space $J^{A}(M, N)$ is identified with $J^{r}(M, N)$.

Proof. For any $M \in \operatorname{Obj}\left(\mathcal{M} f_{m}\right)$, consider the bundle functor $G_{M}: \mathcal{M} f \rightarrow$ $\mathcal{F} \mathcal{M}$ defined by $G_{M} N=J^{r}(M, N)$ on objects and by $G_{M} h=J^{r}\left(\mathrm{id}_{M}, h\right)$ on morphisms.

The assertion $(a)$ implies

$$
J^{r}(M, N)=G_{M} N=P^{r} N\left[\left(T_{k}^{r *} M\right)^{n}, \ell_{M}\right]=P^{r} N\left[\left(T^{A *} M\right)^{n}, \ell_{M}\right],
$$

where $\ell_{M}: G_{n}^{r} \times\left(T_{k}^{r *} M\right)^{n} \rightarrow\left(T_{k}^{r *} M\right)^{n}$ is defined by $\ell_{M}\left(j_{0}^{r} g,\left(T_{k}^{r}\right)_{x} f\right)=$ $\left(T_{k}^{r}\right)_{0} g \circ\left(T_{k}^{r}\right)_{x} f=T_{0}^{A} g \circ T_{x}^{A} f$. It follows $J^{r}(M, N)=J^{A}(M, N)$. For morphisms, we have $J^{A}(g, h)\left(T_{x}^{A} f\right)=T_{g(x)}^{A}\left(h \circ f \circ g^{-1}\right)=j_{g(x)}^{r}\left(h \circ f \circ g^{-1}\right)=$ $J^{r}(g, h)\left(j_{x}^{r} f\right)$ if we denote by $g^{-1}$ the inverse to $g$ considered near $x$.

\section{Bundles of $(p, A)$-COvelocities ANd $(p, A)$-Jets}

Let $\hat{p}: G_{k}^{r} \rightarrow G^{A}$ be a $G_{A}$-stabilizing Lie group homomorphism and $p: G_{k}^{r} \rightarrow$ $\operatorname{Aut}(A)$ its corestriction in the sense of (1.3). For $m \geq k$, we define and investigate the spaces $T_{p}^{A *} M$ of $(p, A)$-covelocities. 
For the present, suppose $m=k$. Consider the left action $\ell$ of $G^{A}$ with its effective left action $\bar{\ell}$ of Aut $A$ on $T_{x}^{A} M$

$$
\ell\left(j_{0}^{r} g, j^{A} \varphi_{x}\right)=\bar{\ell}\left(\pi\left(j_{0}^{r} g\right), j^{A} \varphi_{x}\right)=j^{A} \varphi_{x} \circ\left(j_{0}^{r} g\right)^{-1}
$$

if $\pi: G^{A} \rightarrow$ Aut $A$ denotes the projection Lie group homomorphism. For a Lie subgroup $H \subseteq$ Aut $A$, let us denote by $\operatorname{Orb}\left(H, j^{A} \varphi_{x}\right)$ the $H$-orbit of $j^{A} \varphi_{x}$ with respect to $\bar{\ell}$ restricted to $H \times T_{x}^{A} M$. Since the elements of Aut $A$ determine natural tranformations over $T^{A} M$ determined by (2.1), the values of $T_{x}^{A} f$ on the whole $\operatorname{Orb}$ (Aut $\left.A, j^{A} \varphi_{x}\right)$ are determined by the value $T_{x}^{A} f\left(j^{A} \varphi_{x}\right)$. Indeed, we have

$$
\begin{aligned}
T_{x}^{A} f \circ \ell\left(j_{0}^{r} g, j^{A} \varphi_{x}\right) & =T_{x}^{A} f \circ \bar{\ell}\left(\pi\left(j_{0}^{r} g\right), j^{A} \varphi_{x}\right)= \\
& =\bar{\ell}\left(\pi\left(j_{0}^{r} g\right), T_{x}^{A} f\left(j^{A} \varphi_{x}\right)\right)=\ell\left(j_{0}^{r} g, T_{x}^{A} f\left(j^{A} \varphi_{x}\right)\right) .
\end{aligned}
$$

We are searching for the greatest subgroup $H \subseteq$ Aut $A$ satisfying the existence of a map $X: \operatorname{reg} T_{x}^{A} M \rightarrow N_{A}$ prolongating for $j^{A} \varphi_{x} \in \operatorname{reg} T_{x}^{A} M$ the restrictions $T_{x}^{A} f_{\mid \operatorname{Orb}\left(H, j^{A} \varphi_{x}\right)}$ in the sense of the following formula

$$
\left.X\left(j^{A} \psi_{x}\right)=T_{x}^{A} \hat{f}\left(j^{A} \psi_{x}\right)=T_{x}^{A} f\left(j^{A} \varphi_{x}\right) \circ p\left(\left(j_{0}^{r} \hat{\varphi}_{x}\right)^{-1} \circ j_{0}^{r} \hat{\psi}_{x}\right)\right)
$$

for suitable $T_{x}^{A} \hat{f} \in T_{x}^{A *} M$ and any $j_{0}^{r} \hat{\varphi}_{x} \in j^{A} \varphi_{x}$ and $j_{0}^{r} \widehat{\psi}_{x} \in j^{A} \psi_{x}$. It is easy to see that

$$
j^{A} \varphi_{x, 1}=j^{A} \varphi_{x, 2} \text { implies }\left(j_{0}^{r} \varphi_{x, 1}\right)^{-1} \circ j_{0}^{r} \varphi_{x, 2} \in G_{A}
$$

and thus, the formula (2.3) is correct.

It follows from (2.2) and (2.3) that $H \subseteq p\left(G_{k}^{r}\right)$. Insisting on the condition $H=p\left(G_{k}^{r}\right)$, one deduces easily that (2.3) works only if an additional assumption concerning $p$ is satisfied. It reads as follows

$$
\pi \circ(\hat{p} \circ \hat{p})\left(j_{0}^{r} g\right)=p\left(j_{0}^{r} g\right) \text { for any } \hat{p} \in p \text {. }
$$

We remark that this case leads to the concept of a $p$-vertical $A$-covelocity, which was presented in [16].

Coming back to $(p, A)$-covelocities, we define the Lie subgroups

$$
\begin{aligned}
& \bar{G}_{k}^{r, p}=\left\{j_{0}^{r} g \in G_{k}^{r}, \pi \circ(\hat{p} \circ \hat{p})\left(j_{0}^{r} g\right)=p\left(j_{0}^{r} g\right) \text { for any } \hat{p} \in p\right\}, \\
& G_{k}^{r, p}=\bar{G}_{k}^{r, p} \cap p\left(G_{k}^{r}\right)
\end{aligned}
$$

of $G_{k}^{r}$ determined by $p: G_{k}^{r} \rightarrow$ Aut $A$ under discussion. We put $H=G_{k}^{r, p}$.

On the other hand, a $(p, A)$-covelocity will be a map $X: \operatorname{reg} T_{x}^{A} M \rightarrow N_{A}$ sharing the same value with some $A$-covelocity over a selected element from $\operatorname{reg} T_{x}^{A} M$ and uniquely extended to the whole $\operatorname{reg} T_{x}^{A} M$ by (2.3). We give a definition connecting both of those approaches.

Definition 1. Let $A=\mathbb{D}_{k}^{r} / I, p: G_{k}^{r} \rightarrow$ Aut $A$ be a Lie group homomorphism and $k=m=\operatorname{dim} M$. A map $X: \operatorname{reg} T_{x}^{A} M \rightarrow T_{0}^{A} \mathbb{R}=N_{A}$ is said to be a 
( $p, A)$-covelocity at $x \in M$, i. e., an element of $\left(T_{p}^{A *}\right)_{x} M$ if some of the following conditions is satisfied

(i) for every $j^{A} \varphi_{x} \in \operatorname{reg} T_{x}^{A} M$, there is a covelocity $T_{x}^{A} f \in T_{x}^{A *} M$ satisfying $X\left(j^{A} \varphi_{x}\right)=T_{x}^{A} f\left(j^{A} \varphi_{x}\right)$ and for any $j^{A} \psi_{x} \in \operatorname{reg} T_{x}^{A} M$, the value $X\left(j^{A} \psi_{x}\right)$ is obtained from $X\left(j^{A} \varphi_{x}\right)$ by the following extension

$$
\begin{array}{r}
X\left(j^{A} \psi_{x}\right)=X\left(j^{A} \varphi_{x}\right) \circ p\left(\left(j_{0}^{r} \widehat{\varphi}_{x}\right)^{-1} \circ j_{0}^{r} \widehat{\psi}_{x}\right), j_{0}^{r} \widehat{\varphi}_{x} \in j^{A} \varphi_{x} \\
\quad \text { and } j_{0}^{r} \hat{\psi}_{x} \in j^{A} \psi_{x} .
\end{array}
$$

(ii) for every $j^{A} \varphi_{x} \in \operatorname{reg} T_{x}^{A} M$, there is $T_{x}^{A} f \in T_{x}^{A *} M$ such that the restrictions of $X$ and $T_{x}^{A} f$ to $\operatorname{Orb}\left(G_{k}^{r, p}, j^{A} \varphi_{x}\right)$ coincide and the extensions of $X$ to other $G_{k}^{r, p}$-orbits of reg $T_{x}^{A} M$ are obtained by (2.7).

Then, $T_{p}^{A *} M=\cup_{x \in M}\left(T_{p}^{A *}\right)_{x} M$ is said to be the space of $(p, A)$-covelocities on $M$. For a local diffeomorphism $g: M \rightarrow N$, there is a map $T_{p}^{A *} g: T_{p}^{A *} M \rightarrow$ $T_{p}^{A *} N$ defined as follows

$$
\left(T_{p}^{A *}\right) g(X)=X \circ\left(T_{x}^{A} g\right)^{-1} \text { for } X \in\left(T_{p}^{A *}\right)_{x} M
$$

We note that (2.4) implies the correctness of (2.7) in the sense of its independency on the choice of $j_{0}^{r} \widehat{\varphi}_{x} \in j^{A} \varphi_{x}$ and $j_{0}^{r} \widehat{\psi}_{x} \in j^{A} \psi_{x}$.

It is easy to see that for any $p: G_{k}^{r} \rightarrow$ Aut $A$ satisfying the assumption (2.5), $p$-vertical $A$-covelocities coincide with more general objects of $(p, A)$-covelocities. In other cases, the difference corresponds to the difference between (2.1) and (2.7), namely in the transformations of values within $p\left(G_{k}^{r}\right)$-orbits. One can consider the substitution of $p\left(j_{0}^{r} g\right)=p\left(\left(j_{0}^{r} \varphi_{x}\right)^{-1} \circ j_{0}^{r} \psi_{x}\right)$ for $j_{0}^{r} g=\left(j_{0}^{r} \varphi_{x}\right)^{-1} \circ j_{0}^{r} \psi_{x}$ in (2.1) as an addition of a deformation within orbits expressed by $p$.

Proposition 1. For $m=k=\operatorname{width}(A)$, the system of spaces $T_{p}^{A *} M$ with $T_{p}^{A *}$ maps forms the structure of a natural bundle $P^{r} M\left[N_{A}, \ell\right]$ with the standard fiber formed by $N_{A}$. The left action $\ell: G_{m}^{r} \times N_{A} \rightarrow N_{A}$ is defined as follows

$$
\left.\ell\left(j_{0}^{r} g, j^{A} \alpha\right)=p_{A} \circ r\left(j_{0}^{r} \alpha,\left(p\left(j_{0}^{r} g\right)\right)^{-1}\right)=j^{A} \alpha \circ\left(p\left(j_{0}^{r} g\right)\right)^{-1}\right)
$$

where $r$ is obtained in the obvious way from the standard right action of $G_{m}^{r}$ on $P_{x}^{r} M$ considered on $M_{x}=\mathbb{R}_{0}^{m}$. Further, $p_{A}: \mathbb{D}_{m}^{r} \rightarrow A$ denotes the projection homomorphism of Weil algebras.

Proof. The identification $H: T_{p}^{A *} M \rightarrow P^{r} M\left[N_{A}, \ell\right]$ is given by the assignement $X \mapsto\left\{j_{0}^{r} \alpha_{x}, X\left(j_{0}^{r} \alpha_{x}\right)\right\}$ and $H^{-1}$ by the assignement $\left\{j_{0}^{r} \alpha_{x}, j^{A} \eta\right\} \mapsto\left(j_{0}^{r} \alpha_{x} \mapsto\right.$ $j^{A} \eta$ ). We must verify that the map $H$ is well defined. Indeed, we have

$$
\begin{aligned}
\left\{j_{0}^{r} \alpha_{x} \circ j_{0}^{r} g, X\left(j_{0}^{r} \alpha_{x} \circ j_{0}^{r} g\right)\right\} & =\left\{j_{0}^{r} \alpha_{x} \circ j_{0}^{r} g, X\left(j_{0}^{r} \alpha_{x}\right) \circ p\left(j_{0}^{r} g\right)\right\} \\
& =\left\{r\left(j_{0}^{r} \alpha_{x}, j_{0}^{r} g\right), \ell\left(\left(j_{0}^{r} g\right)^{-1}, X\left(j_{0}^{r} \alpha_{x}\right)\right)\right\} .
\end{aligned}
$$


Clearly, $\left\{j_{0}^{r} \alpha_{x}, j^{A} \eta\right\}$ and $\left\{j_{0}^{r} \alpha_{x} \circ j_{0}^{r} g, j^{A} \eta \circ p\left(j_{0}^{r} g\right)\right\}$ determine the same $(p, A)$ covelocity by its definition.

Furthermore, morphisms of associated bundles $\left\{P^{r} g, \mathrm{id}_{N_{A}}\right\}$ are identified with maps $T_{p}^{A *} g$ since every $\left\{j_{0}^{r} \alpha_{x}, T_{x}^{A} f\left(j_{0}^{r} \alpha_{x}\right)\right\}$ is assigned $\left\{j_{0}^{r}\left(g \circ \alpha_{x}\right), T_{x}^{A} f\left(j_{0}^{r} \alpha_{x}\right)\right\}=$ $\left.\left\{j_{0}^{r}\left(g \circ \alpha_{x}\right), T_{x}^{A} f \circ\left(T_{x}^{A} g\right)^{-1}\right)\left(j_{0}^{r}\left(g \circ \alpha_{x}\right)\right)\right\}$. This completes the proof.

There is an easy extension of the result from $m=k=$ width $A$ to the cases of $m>k$. We can replace $A=\mathbb{D}_{k}^{r} / I$ by $\tilde{A}=\mathbb{D}_{m}^{r} /\left(I \vee\left\langle\tau_{k+1}, \ldots, \tau_{m}\right\rangle\right)$ adding formally $m-k$ indeterminates. As for the subgroups $G^{A}$ or $G_{A} \subseteq G_{k}^{r}$, replace them by $G^{\tilde{A}}$ and $G_{\tilde{A}} \subset G_{m}^{r}$ as follows. For any $j_{0}^{r} \tilde{g} \in G_{m}^{r}$, put

$$
\begin{aligned}
& j_{0}^{r} \tilde{g} \in G^{\tilde{A}} \text { or } j_{0}^{r} \tilde{g} \in G_{\tilde{A}} \text { if and only if } \operatorname{pr}_{1} \circ j_{0}^{r} \tilde{g} \circ j_{0}^{r} i_{1} \in G^{A} \\
& \quad \text { or } \operatorname{pr}_{1} \circ j_{0}^{r} \tilde{g} \circ j_{0}^{r} i_{1} \in G_{A} \text { and } \operatorname{pr}_{2} \circ j_{0}^{r} \tilde{g} \circ j_{0}^{r} i_{1}=j_{0}^{r} 0^{m-k}
\end{aligned}
$$

for the zero function on $\mathbb{R}^{k}$, the inclusion $i_{1}: \mathbb{R}^{k} \rightarrow \mathbb{R}^{m}$ defined by $\left(x^{1}, \ldots, x^{k}\right) \mapsto$ $\left(x^{1}, \ldots, x^{k}, 0, \ldots, 0\right)$ and the projection $\mathrm{pr}_{2}: \mathbb{R}^{k} \times \mathbb{R}^{m-k} \rightarrow \mathbb{R}^{m-k}$.

Let us replace $p: G_{k}^{r} \rightarrow \operatorname{Aut}(A)$ by a Lie group homomorphism $\tilde{p}: G_{m}^{r} \rightarrow$ $\operatorname{Aut}(\tilde{A})$. Consider any free basis $\mathscr{B}_{1}$ of generators of $G_{k}^{r} \simeq G_{k}^{r} \times\left\{j_{0}^{r} \operatorname{id}_{\mathbb{R}^{m-k}}\right\} \subseteq G_{m}^{r}$ and complete it to a free basis of generators $\mathscr{B}$ of $G_{m}^{r}$. Then, we define $\tilde{p}: G_{m}^{r} \rightarrow$ $\operatorname{Aut}(\tilde{A})$ by $j_{0}^{r} g \times\left\{j_{0}^{r} \operatorname{id}_{\mathbb{R}^{m-k}}\right\} \mapsto p\left(j_{0}^{r} g\right)$ for $j_{0}^{r} g \in G_{k}^{r}$ and by $j_{0}^{r} \tilde{g} \mapsto j_{0}^{r} \operatorname{id}_{\mathbb{R}^{m}}$ in case of $j_{0}^{r} \tilde{g} \in \mathcal{B}-\mathscr{B}_{1}$. Further, the left action from (2.9) is replaced by $\ell$ : $G_{m}^{r} \times N_{\tilde{A}} \rightarrow N_{\tilde{A}}$, which is defined as follows

$$
\begin{aligned}
\ell\left(j_{0}^{r} g, j^{A} \alpha\right) & =\ell\left(j_{0}^{r} g, j^{\tilde{A}} \alpha\right) \\
& =p_{\tilde{A}} \circ r\left(j_{0}^{r} \alpha,\left(\tilde{p}\left(j_{0}^{r} g\right)\right)^{-1}\right) \\
& \left.=j^{\tilde{A}} \alpha \circ\left(\tilde{p}\left(j_{0}^{r} g\right)\right)^{-1}\right) .
\end{aligned}
$$

So, we can modify the extension of $(p, A)$-covelocity $X$ from $j^{A} \varphi_{x} \in \operatorname{reg} T_{x}^{A} M$ to another value $j^{A} \psi_{x} \in \operatorname{reg} T_{x}^{A} M$ from (2.7) as follows

$$
X\left(j^{A} \psi_{x}\right)=X\left(j^{A} \varphi_{x}\right) \circ\left(\tilde{p}\left(j_{0}^{r}\left(\hat{\psi}_{x}^{-1} \circ \widehat{\varphi}_{x}\right)\right)^{-1}\right.
$$

where $j_{0}^{r} \widehat{\varphi}_{x}$ and $j_{0}^{r} \widehat{\psi}_{x}$ are formally considered as arbitrary elements of $j^{\tilde{A}} \varphi_{x}$ and $j^{\tilde{A}} \psi_{x} \in \operatorname{reg} T_{x}^{\tilde{A}} M$. Since the definition (2.8) of $T_{p}^{A *}$-maps remains unchanged, we can reformulate Proposition 1 as follows

Proposition 2. For $m \geq k$ and a Lie group homomorphism $p: G_{k}^{r} \rightarrow \operatorname{Aut}(A)$, the system of spaces $T_{p}^{A *} M$ of $(p, A)$-covelocities with their $T_{p}^{A *}{ }^{*}$-maps is identified with the system of spaces $T_{\tilde{p}}^{\tilde{A} *} M$ with their $T_{\tilde{p}}^{\tilde{A} *}$-maps and consequentely to a natural bundle $T_{p}^{A *} M=P^{r} M\left[N_{A}, \ell\right]$ with the standard fiber formed by $N_{A} \simeq$ $N_{A} \vee\left\langle\tau_{k+1}, \ldots, \tau_{m}\right\rangle$ and the left action $\ell$ defined by (2.12). 
Let us define the space $J_{p}^{A}(M, N)$ of $(p, A)$-jets.

Definition 2. Let $A=\mathbb{D}_{k}^{r} / I$ and $p: G_{k}^{r} \rightarrow \operatorname{Aut}(A)$ be as above, $m \geq k=$ $\operatorname{width}(A)$. For $(M, N) \in \operatorname{Obj}\left(\mathcal{M} f_{m}\right) \times \operatorname{Obj}(\mathcal{M} f)$, define $\left(J_{p}^{A}\right)_{x}(M, N)_{y}=\{X:$ $\operatorname{reg} T_{x}^{A} M \rightarrow T_{y}^{A} N$; for all $j^{A} \varphi_{x} \in \operatorname{reg} T_{x}^{A} M$, there is $T_{x}^{A} f \in J_{x}^{A}(M, N)_{y}$ satisfy$\operatorname{ing} X\left(j^{A} \varphi_{x}\right)=T_{x}^{A} f\left(j^{A} \varphi_{x}\right)$ and $X\left(j^{A} \psi_{x}\right)=X\left(j^{A} \varphi_{x}\right) \circ \tilde{p}\left(\left(j_{0}^{r} \hat{\varphi}_{x}\right)^{-1} \circ j_{0}^{r} \hat{\psi}_{x}\right)$ for any $\left.j_{0}^{r} \widehat{\varphi}_{x} \in j^{\tilde{A}} \varphi_{x}, j_{0}^{r} \hat{\psi}_{x} \in j^{\tilde{A}} \psi_{x}\right\}$. We define the space $J_{p}^{A}(M, N)$ of $(p, A)$-jets setting

$$
J_{p}^{A}(M, N):=\bigcup_{x \in M, y \in N}\left(J_{p}^{A}\right)_{x}(M, N)_{y} .
$$

For a local diffeomorphism $g: M_{1} \rightarrow M_{2}$ and a smooth map $h: N_{1} \rightarrow N_{2}$, define the map $J^{A}(g, h): J_{p}^{A}\left(M_{1}, N_{1}\right) \rightarrow J_{p}^{A}\left(M_{2}, N_{2}\right)$ by

$$
J^{A}(g, h)(X)=T_{f(x)}^{A} h \circ X \circ\left(T_{x}^{A} g\right)^{-1}
$$

for all $X \in\left(J_{p}^{A}\right)_{x}(M, N)_{y}$.

In the very end, we give the geometrical description of the spaces $J^{A}(M, N)$. Denote by $\mathcal{N} \mathscr{E}(A)$ the group of natural equivalences over $T^{A}$ identified with the group $\operatorname{Aut}(A)$.

Proposition 3 (Kolář and Mikulski [10]). Every bundle functor $F$ defined on the product category $\mathcal{M} f_{m} \times \mathcal{M} f$ of order $r$ in the first factor and preserving products in the second factor is of the form $F(M, N)=P^{r} M\left[G^{F} N, H_{N}^{F}\right]=P^{r} M\left[T^{A} N, H_{N}^{F}\right]$ for a product preserving bundle functor $G^{F}=T^{A}$ defined on objects by $G^{F} N=$ $F_{0}\left(\mathbb{R}^{m}, N\right)=T^{A} N$, on morphisms by $G^{F} f=F_{0}\left(\mathrm{id}_{\mathbb{R}^{m}}, f\right)=T^{A} f$ and for a Lie group homomorphism $H^{F}: G_{m}^{r} \rightarrow \mathcal{N} \mathscr{E}(A) \simeq \operatorname{Aut}(A)$ defined by $H_{N}^{F}\left(j_{0}^{r} g\right)(a)=$ $F_{0}\left(g, \operatorname{id}_{N}\right)(a)$ for every $a \in G^{F} N \simeq T^{A} N$.

Proposition 4. For $m \geq k$, the spaces $J_{p}^{A}(M, N)$ with $J_{p}^{A}$-maps form a bundle functor on the product category $\mathcal{M} f_{m} \times \mathcal{M} f$ satisfying $J_{p}^{A}(M, \mathbb{R})_{0}=T_{p}^{A *} M$ and $J_{p}^{A}\left(g, \mathrm{id}_{\mathbb{R}}\right){ }_{\mid \operatorname{Obj}\left(\mathcal{M} f_{m}\right) \times\left\{\mathbb{R}_{0}\right\}}=T_{p}^{A *} g$. Moreover, $J_{p}^{A}$ preserves products in the second factor and $J_{p}^{A}(M, N) \simeq P^{r} M\left[G^{F} N, H_{N}^{F}\right]$ for $G^{F}=T^{A}$ and $H^{F} \simeq \ell$ for $\ell$ defined by (2.9) or (2.12).

Proof. We are searching for a bundle functor of the required kind on $J_{p}^{A}$ extending $T_{p}^{A *}$. We prove

$$
J_{p}^{A}(M, N) \simeq P^{r} N\left[\left(T_{p}^{A *} M\right)^{n}, \bar{\ell}\right] \rightarrow M \times N,
$$

where $\bar{\ell}: G_{n}^{r} \times\left(T_{p}^{A *} M\right)^{n} \rightarrow\left(T_{p}^{A *} M\right)^{n}$ is defined in the following way. Let us identify $\left(\left\{j_{0}^{r} \alpha_{x}^{1}, j^{A} \theta^{1}\right\}, \ldots,\left\{j_{0}^{r} \alpha_{x}^{n}, j^{A} \theta^{n}\right\}\right) \in\left(T_{p}^{A *} M\right)_{x}^{n} \simeq\left(P_{x}^{r} M\left[N_{A}, \ell\right]\right)^{n}$ with $\left\{j_{0}^{r} \alpha_{x}, j^{A} \eta^{1}, \ldots, j^{A} \eta^{n}\right\} \in P_{x}^{r} M \times_{G_{m}^{r}} N_{A}^{n}$ where $j_{0}^{r} \alpha_{x} \in P_{x}^{r} M$ is arbitrary and 
$j^{A} \eta^{1}, \ldots, j^{A} \eta^{n} \in N_{A}$ are obtained by $j^{A} \eta^{i}=\ell\left(j_{0}^{r} h_{i}^{-1}, j^{A} \theta^{i}\right)$ for $j_{0}^{r} h_{i} \in G_{m}^{r}$ satisfying $j_{0}^{r} \alpha_{x}=r\left(j_{0}^{r} \alpha_{x}^{i}, j_{0}^{r} h_{i}\right)$. In this identification, we put

$$
\begin{aligned}
& \bar{\ell}\left(j_{0}^{r} g,\left\{j_{0}^{r} \alpha_{x}, j^{A} \eta^{1}, \ldots, j^{A} \eta^{n}\right\}\right)= \\
& \quad=\left\{j_{0}^{r} \alpha_{x}, T_{0}^{A} g^{1}\left(j^{A} \eta^{1}, \ldots, j^{A} \eta^{n}\right), \ldots, T_{0}^{A} g^{n}\left(j^{A} \eta^{1}, \ldots, j^{A} \eta^{n}\right)\right\} .
\end{aligned}
$$

It is clear that, for any $X \in J_{x}^{A}(M, N)_{y}$ and $j_{0}^{r} \gamma_{y} \in P_{y}^{r} N$, we have $\left(T_{0}^{A} \gamma_{y}\right)^{-1} X \in$ $\left(\left(T_{p}^{A *}\right)_{x} M\right)^{n}$. Thus, $X \simeq\left\{j_{0}^{r} \gamma_{y},\left(T_{0}^{A} \gamma_{y}\right)^{-1}(X)\right\} \in P_{y}^{r} N \times_{G_{n}^{r}}\left(\left(T_{p}^{A *}\right)_{x} M\right)^{n}$ with respect to the standard right action of $G_{n}^{r}$ on $P^{r} N$ and the left action $\bar{\ell}$ of $G_{n}^{r}$ on $\left(T_{p}^{A *} M\right)^{n}$ given by the composition of maps $T_{0}^{A} g: T_{0}^{A} \mathbb{R}^{n} \rightarrow T_{0}^{A} \mathbb{R}^{n}$ determined by $j_{0}^{r} g \in G_{n}^{r}$ with elements of $\left(T_{p}^{A *} M\right)^{n}$, which can be also expressed by (2.14).

One can observe easily the smoothness of $\bar{\ell}$ from (2.14). Further, there is the obvious projection $P^{r} N\left[\left(T_{p}^{A *} M\right)^{n}, \bar{\ell}\right] \rightarrow M \times N$ compatible with that of $J_{p}^{A}(M, N) \rightarrow$ $M \times N$, which proves its smoothness.

The maps $J_{p}^{A}(g, h)$ coincide with the compositions of the pairs of smooth maps $J_{p}^{A}\left(\operatorname{id}_{M}, h\right) \simeq\left\{P^{r} h, \operatorname{id}_{\left(T_{D}^{A *} M\right)^{n}}\right\}$ and $J_{p}^{A}\left(g, \operatorname{id}_{N}\right) \simeq\left\{\operatorname{id}_{P^{r} N}, T_{p}^{A *} g\right\}$. The verification of the localization condition from the definition of a bundle functor (see [9]) is easy. The last assertion is a direct consequence of Proposition 3.

\section{REFERENCES}

[1] J. R. Alonso, "Jet manifolds associated to a Weil bundle," Arch. Math. Brno, vol. 36, pp. 195-209, 2000.

[2] R. J. Alonso-Blanco and D. Blázquez-Sanz, "The only global contact transformations of order two or more are point transformations," Journal of Lie Theory, vol. 15, pp. 135-143, 2005.

[3] W. Bertram, "Differential geometry over general base fields and rings," to appear in the Memoirs of AMS.

[4] W. Bertram, H. Glockner, and K. H. Neeb, "Differential calculus over general base fields and rings," Expo Math., vol. 22, pp. 213-282, 2004.

[5] G. N. Bushueva, "Weil functors and product preserving functors on the category of parameterdependent manifolds," Russian Mathematics (Izvestia VUZ. Matematika), vol. 5, pp. 14-21, 2005.

[6] D. J. Eck, "Product-preserving functors on smooth manifolds," J. Pure Appl. Algebra, vol. 6, no. 42, pp. 133-140, 1986.

[7] G. Kainz and P. W. Michor, "Natural transformations in differential geometry," Czechosl. Math. J., vol. 37, pp. 584-607, 1987.

[8] I. Kolář, "Covariant approach to natural transformations of Weil bundles," Comment. Math. Univ. Carolinae, pp. 99-105, 1986.

[9] I. Kolář, P. W. Michor, and J. Slovák, Natural Operations in Differential Geometry. Springer Verlag, 1993.

[10] I. Kolář and W. M. Mikulski, "On the fiber product preserving bundle functors," Diff. Geom. Appl., vol. 11, pp. 105-111, 1999.

[11] M. Kureš, "Weil algebras associated to functors of third order semiholonomic velocities," to appear in Math. J. of Okayama University.

[12] F. J. Muriel, J. Muñoz, and J. Rodriguez, "Weil bundles and jet spaces," Arch. Math. Brno, vol. 50, pp. 721-748, 2000. 
[13] H. Nishimura, "Axiomatic Differential Geometry I - Towards model categories of differential geometry," to appear in Math. for Applications, Brno.

[14] V. V. Shurygin, "Manifolds over algebras and their application to the geometry of jet bundles," Russian Math. Surveys, vol. 48, pp. 75-104, 1993.

[15] V. V. Shurygin, Jr., "Radiance obstructions for smooth manifolds over Weil algebras," Russian Mathematics (Izvestia VUZ. Matematika), vol. 49, no. 5, pp. 67-79, 2005.

[16] J. Tomáš, "On bundles of covelocities," Lobachevskii J. of Math., vol. 30, no. 4, pp. 280-289, 2009.

\section{Author's address}

\section{Jiří Tomáš}

Brno University of Technology, Faculty of Chemistry, Department of Physical and Applied Chemistry, Purkyňova 118, 61200 Brno, Czech Republic

E-mail address: Tomas @ fch. vutbr.cz 